\title{
Import of multidrug-resistant bacteria from abroad through interhospital transfers, Finland, 2010-2019
}

Mikael Kajova ${ }^{1,2}$, Tamim Khawaja ${ }^{1,2}$, Jonas Kangas ${ }^{1,2}$, Hilda Mäkinen ${ }^{1,2}$, Anu Kantele ${ }^{1,3}$

1. Inflammation Center, Department of Infectious Diseases, University of Helsinki and Helsinki University Hospital, Helsinki, Finland

2. Meilahti Infectious Diseases and Vaccine Research Center, MeiVac, University of Helsinki and Helsinki University Hospital, Helsinki, Finland

3. Human Microbiome Research Program, Faculty of Medicine, University of Helsinki, Helsinki, Finland

Correspondence: Anu Kantele (anu.kantele@hus.fi)

Citation style for this article:

Kajova Mikael, Khawaja Tamim, Kangas Jonas, Mäkinen Hilda, Kantele Anu. Import of multidrug-resistant bacteria from abroad through interhospital transfers, Finland, 2010-2019. Euro Surveill. 2021;26(39): pii=2001360. https://doi.org/10.2807/1560-7917.ES.2021.26.39.2001360

Background: While $20-80 \%$ of regular visitors to (sub)tropical regions become colonised by extended-spectrum $\beta$-lactamaseproducing Enterobacteriaceae (ESBL-PE), those hospitalised abroad often also carry other multidrugresistant (MDR) bacteria on return; the rates are presumed to be highest for interhospital transfers. Aim: This observational study assessed MDR bacterial colonisation among patients transferred directly from hospitals abroad to Helsinki University Hospital. We investigated predisposing factors, clinical infections and associated fatalities. Methods: Data were derived from screening and from diagnostic samples collected between 2010 and 2019. Risk factors of colonisation were identified by multivariable analysis. Microbiologically verified symptomatic infections and infection-related mortality were recorded during post-transfer hospitalisation. Results: Colonisation rates proved highest for transfers from Asia (69/96; $71.9 \%)$ and lowest for those within Europe (99/524; $18.9 \%)$. Of all 698 patients, $208(29.8 \%)$ were colonised; among those, $163(78.4 \%)$ carried ESBL-PE, 28 (13.5\%) MDR Acinetobacter species, 25 (12.0\%) meticillin-resistant Staphylococcus aureus, 25 (12.0\%) vancomycin-resistant Enterococcus, 14 (6.7\%) carbapenemase-producing Enterobacteriaceae, and 12 (5.8\%) MDR Pseudomonas aeruginosa; 46 strains tested carbapenemase gene-positive. In multivariable analysis, geographical region, intensive care unit (ICU) treatment and antibiotic use abroad proved to be risk factors for colonisation. Clinical MDR infections, two of them fatal (1.0\%), were recorded for 22 of $208(10.6 \%)$ MDR carriers. Conclusions: Colonisation by MDR bacteria was common among patients transferred from foreign hospitals. Region of hospitalisation, ICU treatment and antibiotic use were identified as predisposing factors. Within 30 days after transfer, MDR colonisation manifested as clinical infection in more than $10 \%$ of the carriers.

\section{Introduction}

The spread of antimicrobial resistance (AMR) is strongly associated with international travel: $20-80 \%$ of visitors to high-risk regions become colonised and carry multidrug-resistant (MDR) bacteria back to their home country [1]. In high-income countries, rising background resistance and, particularly, import of MDR bacteria into hospitals from overseas is a concern.

Compared with infections by bacteria susceptible to antibiotics, infections by resistant bacteria are associated with greater mortality, longer hospitalisation and higher costs $[2,3]$. Colonisation by MDR bacteria, such as extended-spectrum $\beta$-lactamaseproducing Enterobacteriaceae (ESBL-PE), carbapenemase-producing Enterobacteriaceae (CPE), meticillin-resistant Staphylococcus aureus (MRSA), MDR Acinetobacter species (MDRACI), MDR Pseudomonas aeruginosa (MDRPA) and vancomycin-resistant Enterococcus (VRE), often remains asymptomatic but increases the risk of developing an infection. Colonised individuals may spread bacteria to contacts and the broader environment. Recently, we showed that approximately half of all ESBL-PE imported by travellers carry either intestinal or extraintestinal/ uropathogenic virulence genes [4].

Travellers hospitalised abroad are at increased risk of acquiring MDR bacteria [5-13]. In a study by Khawaja et al. in which 1,122 such patients were screened within 12 months after hospitalisation, $55 \%$ of those returning from (sub)tropical areas and $17 \%$ of those returning from temperate zones carried at least one type of MDR bacteria, mostly ESBL-PE [13].

Research into types and rates of travel-acquired MDR bacteria and the associated risk factors aids prioritisation of infection control resources and selection of empirical antibiotics. Interhospital patient transfers from abroad involve an increased risk of MDR bacterial 


\section{FIGURE 1}

Multidrug-resistant bacterial colonisation among patients transferred directly from hospitals abroad to Helsinki University Hospital, Finland, January 2010- June 2019 (n=698)

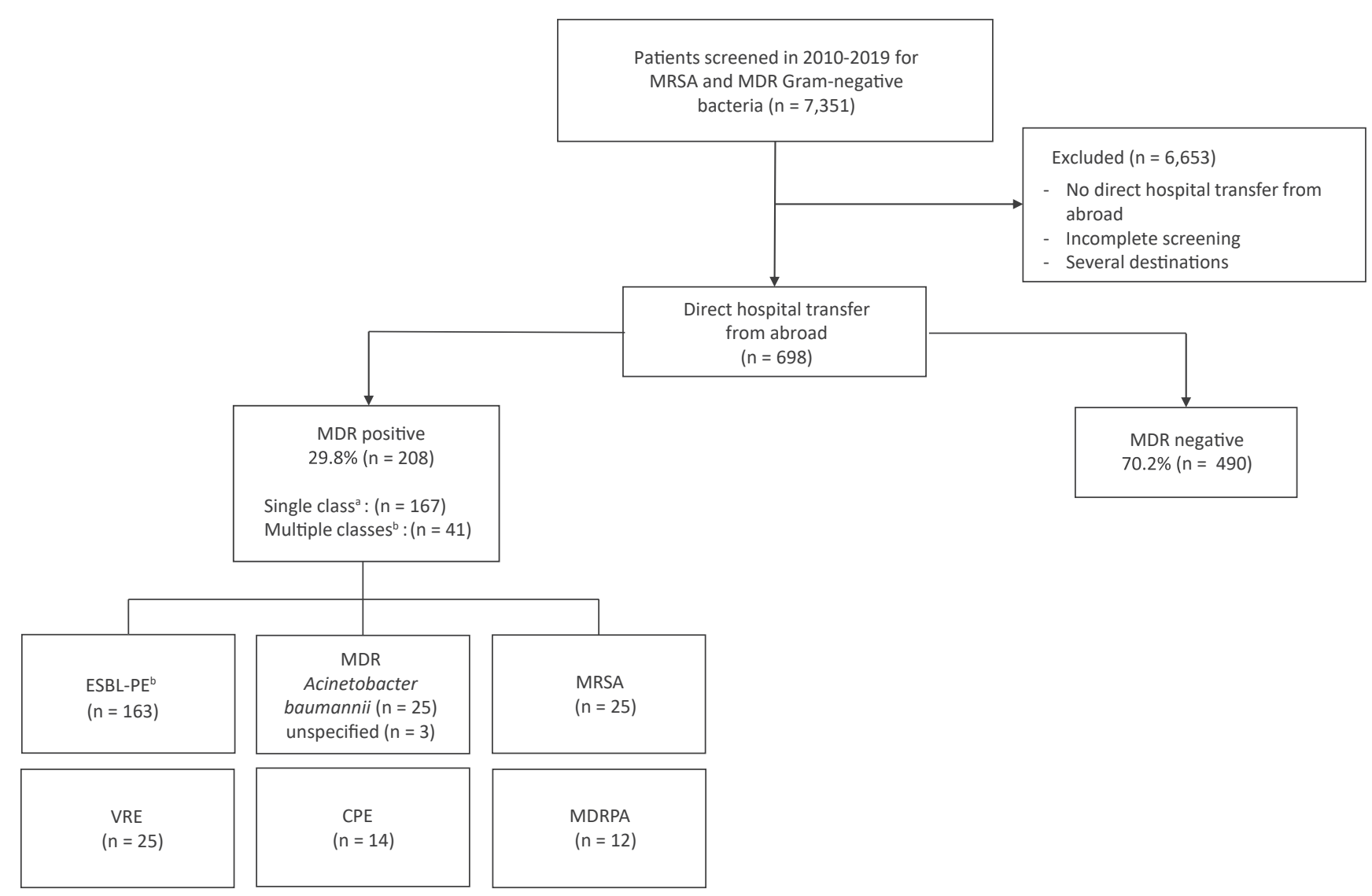

CPE: carbapenemase-producing Enterobacteriaceae; ESBL-PE: extended-spectrum $\beta$-lactamase-producing Enterobacteriaceae; MDR: multidrugresistant; MDRPA: multidrug-resistant Pseudomonas aeruginosa; MRSA: meticillin-resistant Staphylococcus aureus; VRE: vancomycinresistant Enterococcus.

a MDR bacterial classes: CPE, ESBL-PE, MDR Acinetobacter species, MDRPA, MRSA, VRE. A patient denoted with single class had one or more isolates of the same MDR bacterial class, one denoted with multiple classes had isolates of two or more MDR bacterial classes.

b In addition, 10 patients carried non-ESBL Enterobacteriaceae resistant to third-generation cephalosporins.

carriage [13]. Such transfers may pose a substantial threat especially to hospitals in countries with a lower AMR prevalence, yet data on actual rates and risk have thus far been scarce. This study was undertaken to provide region-specific rates intended to provide basis for infection control management when devising guidelines and targeting resources.

\section{Methods}

\section{Study design}

The prevalence and risk factors of colonisation by MDR bacteria were studied among patients transferred from hospitals abroad to Helsinki University Hospital, Finland (HUH) between 1 January 2010 and 30 June 2019. We searched the $\mathrm{HUH}$ electronic infection control database for patients screened for both MRSA and MDR Gram-negative bacteria (MDRGNB). The latter screen comprises detection of CPE, ESBL-PE, MDRACl and
MDRPA. For such patients, electronic medical records were explored and those with interhospital transfer data included. Patients with records from 2010 to 2013 were covered in a previous AMR investigation [13] that did not report separately on direct hospital transfers.

\section{Definitions}

Hospitalisation abroad was defined as a hospital stay of more than $24 \mathrm{~h}$ or an admission involving surgery or some other major invasive procedure. Direct transfer was defined as hospitalisation that continued immediately on return to Finland, with no overnight stay outside hospitals (excluding flights).

\section{Inclusion criteria}

The microbiological inclusion criteria comprised (i) a record of rectal swab or stool sample for MDRGNB screening within 3 days and (ii) MRSA screening from all three sites (nose, throat/trachea, groin/perineum) 
Colonisation by multidrug-resistant bacteria of patients transferred directly from hospitals abroad to Helsinki University Hospital, Finland, 2010-2019 ( $\mathrm{n}=698)$

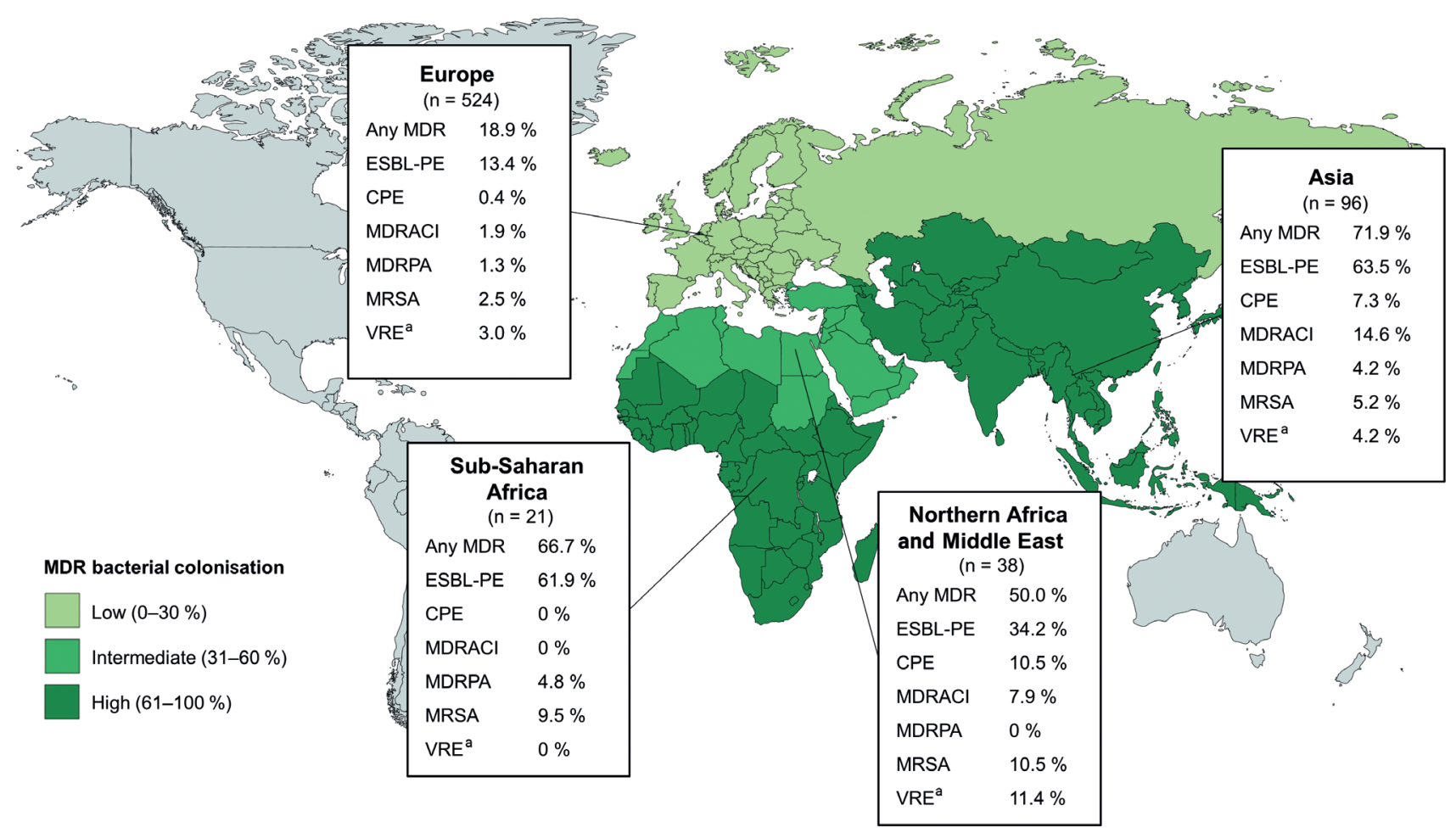

CPE: carbapenemase-producing Enterobacteriaceae; ESBL-PE: extended-spectrum $\beta$-lactamase-producing Enterobacteriaceae; MDR: multidrugresistant; MDRACI: multidrug-resistant Acinetobacter; MDRPA: multidrug-resistant Pseudomonas aeruginosa; MRSA: meticillin-resistant Staphylococcus aureus; VRE: vancomycin-resistant Enterococcus.

Other continents are not shown because of low number of cases: Latin America and the Caribbean $(n=10)$, Northern America $(n=3)$, Oceania $(n=6)$. The government-controlled area of Cyprus is included under Europe.

a VRE \% given of screened patients.

The map was created with mapchart.net.

within 30 days after transfer day. According to HUH guidelines, MDRGNB, MRSA and VRE screening samples are to be taken from all patients transferred directly from hospitals abroad. However, before 2016, this only applied to countries outside the Nordic region. The guidelines also advise sampling of skin lesions, urine from in-dwelling catheters in place for over 7 days, and trachea from intubated/tracheostomy patients. During the study period from 2010 to 2019, some (mostly minor) modifications were made to the guidelines, summarised in the Supplement (Supplementary Table $\mathrm{S} 1)$. Two or three specimens were recommended for each item/area on separate days. A VRE screening was not included as a criterion since it had not been performed regularly, but VRE results were recorded when available. We recorded all findings of MDR bacteria in screening and diagnostic samples collected within 30 days from the first screening.

\section{Exclusion criteria}

Medical records were searched for other documented stays and hospitalisations abroad. Stays outside Europe in regions other than those where hospitalised during the previous 12 months led to exclusion. Since travel within Europe is common and often not recorded in patient files, this was not taken as an exclusion criterion. Patients with multiple foreign hospitalisations were included only if all the hospitalisations took place in the same European country or, for other parts of the world, within the same geographical region.

\section{Data collection}

We collected data on underlying diseases, chronic alcohol abuse, travel-related factors and information linked to hospitalisation (antibiotics, interventions, intensive care unit (ICU) treatment, duration, diagnosis), and calculated the Charlson comorbidity index (CCI) [14]. In addition to documented antibiotic use, records of bacterial infections known to require antibiotics or surgery for which prophylaxis is advised were classified as 'antibiotic use abroad'. Ongoing antibiotic treatments at the time of screening were recorded but marked as negative if at least one set of samples had been taken without any antibiotics administered for $24 \mathrm{~h}$. Because of the complexity of potential antimicrobial effects, we chose not to consider whether or not 
an antibiotic acted against the MDR bacteria carried by the patient.

Symptomatic, microbiologically verified MDR infections and direct mortality caused by such infections were assessed by an infectious diseases specialist; they were recorded from transfer until discharge or for a maximum of 30 days. Because of incomplete data, potential MDR bacterial infections treated abroad were not evaluated.

\section{Microbiological methods}

The various MDR bacteria were identified by the standard methods used in the HUH laboratory HUSLAB, as follows.

MRSA was identified after overnight enrichment on eMRSA broth (Copan Italia, Brescia, Italy) or selective in-house MRSA enrichment broth [15] and subsequent culture on chromID MRSA (bioMérieux, Marcy-l'Étoile, France) or CHROMagar MRSA (CHROMagar, Paris, France), and confirmed by quantitative PCR for the $S$. aureus-specific nuclease and mecA gene [15].

VRE were detected using enrichment Enterococcosel broth (BBL, Cockeysville, MD) followed by culture on in-house selective media, as previously described [15], or CHROMagar VRE media. Positive findings were confirmed by in-house PCR [15].

ESBL-PE and CPE were analysed by plating directly on CHROMagar ESBL and CHROMagar Klebsiella pneumoniae Carbapenemase (KPC) or CHROMagar mSuperCARBA, respectively. Identification of ESBL-PE species was confirmed by matrix-assisted laser desorption ionisation time-of-flight (MALDI-TOF; Vitek-MS, bioMérieux) and resistance was determined according to the guidelines from the Clinical and Laboratory Standards Institute (CLSI) and, from 2011, the European Committee on Antimicrobial Susceptibility testing (EUCAST) [1517]. CPE were confirmed with in-house PCR targeting the carbapenemase gene [15].

MDRACI and MDRPA were screened on ESBL and KPC plates. Cultures were tested by C-390, VITEK-GN or MALDITOF for species identification. Acinetobacter isolates resistant to meropenem and Pseudomonas isolates resistant to both meropenem and ceftazidime were analysed by PCR for carbapenemase genes [15].

\section{Statistical analyses}

We used SPSS v. 25.0 (IBM Corp., Armonk, New York, United States) for all statistical analyses. For univariate analyses the chi-squared test, Fisher's exact test or binary logistic regression were used, as appropriate. For multivariable analysis, variables with a $p$ value below 0.2 in univariate analysis, or those assessed as clinically relevant, were included. In cases of two strongly correlating explanatory variables, only one was chosen. The most parsimonious model was found by backward selection based on Akaike information criteria.

\section{Ethical statement}

The present study was approved by the research board of HUH Department of Internal Medicine. Since this investigation did not involve an intervention, an ethics committee review was not required (Finnish Medical Research Act).

\section{Results}

\section{Study population}

A total of 698 patients undergoing direct hospital transfers between 1 January 2010 and 30 June 2019 met the inclusion criteria (Figure 1). Of the 86 countries where initial hospitalisation occurred, Spain, Estonia and Thailand accounted for 310 patients (44.4\%). Europe was the most common region with 524 patients (75.1\%). The majority of those returning from Asia (69/96; 71.9\%) had been hospitalised in South East Asia. For a list of the countries in which hospitalisation took place, see Supplement (Supplementary Table S2). Residence abroad was recorded as type of travel for 96 patients (13.8\%), visiting friends and relatives (VFR) for $49(7.0 \%)$, and work, holiday and other reasons for the rest $(553 ; 79.2 \%)$.

The median duration of hospitalisation abroad was 9 days (range: $1-643)$. A total of 190 (27.2\%) patients had been admitted to ICU and 290 (41.5\%) had undergone surgery abroad. The primary diagnosis on repatriation was non-trauma disease for 375 (53.7\%) patients and trauma for $323(46.3 \%)$ patients.

Antibiotic use abroad and during screening The medical records included documentation of antibiotic use abroad for 383 patients (54.9\%). After transfer, $458(65.6 \%)$ patients had three-site MRSA and faecal MDRGNB screening at least once without ongoing antibiotic treatment, while $182(26.1 \%)$ had ongoing antibiotic therapy at screenings. For 58 patients, data were missing or antibiotics were used during part of the screenings.

Findings of multidrug-resistant bacteria

A total of 208 patients (29.8\%) were colonised by MDR bacteria, 41 by more than one class of MDR bacteria (Figure 1). ESBL-PE were the most common findings with $163(23.4 \%)$ carriers, 76 of them $(10.9 \%$ of the total study population) showing solely ESBLproducing Escherichia coli. Twenty-eight patients carried MDRACI, 25 carried MRSA, 14 carried CPE and 12 MDRPA, while 25 had VRE; data on VRE screening were missing for 35. Comparisons between the various years did not reveal significant differences in total MDR bacterial carriage rates 2010-2019 (Supplement, Supplementary Table S3). Figure 2 shows the colonisation rates by geographical regions. 
TABLE 1

Patient characteristics and risk factor analyses of multidrug-resistant bacterial colonisation among patients screened after direct transfer from hospitals abroad to Helsinki University Hospital, Finland, 2010-2019 (n=698)

\begin{tabular}{|c|c|c|c|c|c|c|c|c|c|}
\hline & \multirow{2}{*}{$\begin{array}{c}\text { Patients } \\
(\mathrm{n}=698) \\
\mathrm{n}\end{array}$} & \multicolumn{2}{|c|}{$\begin{array}{c}\text { MDR bacteria } \\
\text { positive }(n=208)\end{array}$} & \multicolumn{2}{|c|}{$\begin{array}{c}\text { MDR bacteria } \\
\text { negative }(n=490)\end{array}$} & \multirow{2}{*}{$\begin{array}{c}\text { OR }(95 \% \mathrm{Cl}) \\
\text { in univariate } \\
\text { analysis }\end{array}$} & \multirow{2}{*}{$\begin{array}{c}\mathrm{p} \text { value in } \\
\text { univariate } \\
\text { analysis }\end{array}$} & \multirow{2}{*}{$\begin{array}{c}\text { AOR }(95 \% \mathrm{Cl}) \\
\text { in multivariable } \\
\text { analysis }^{\mathrm{a}}\end{array}$} & \multirow{2}{*}{$\begin{array}{l}\mathrm{p} \text { value in } \\
\text { multivariable } \\
\text { analysis }^{\mathrm{a}}\end{array}$} \\
\hline & & $\mathrm{n}$ & $\%$ & $\mathrm{n}$ & $\%$ & & & & \\
\hline \multicolumn{10}{|l|}{ Sex } \\
\hline Male & 427 & 134 & 31.4 & 293 & 68.6 & $1.2(0.9-1.7)$ & 0.25 & $\mathrm{NI}$ & $\mathrm{NI}$ \\
\hline Female & 271 & 74 & 27.3 & 197 & 72.7 & Ref & Ref & $\mathrm{NI}$ & $\mathrm{NI}$ \\
\hline \multicolumn{7}{|l|}{ Age groups (years) } & 0.55 & $\mathrm{NA}$ & $\mathrm{NA}$ \\
\hline $0-5$ & 27 & 4 & 14.8 & 23 & 85.2 & Ref & Ref & $\mathrm{NI}$ & $\mathrm{NI}$ \\
\hline $6-17$ & 35 & 9 & 25.7 & 26 & 74.3 & $2.0(0.5-7.3)$ & 0.30 & $\mathrm{NI}$ & $\mathrm{NI}$ \\
\hline $18-30$ & 61 & 19 & 31.1 & 42 & 68.9 & $2.6(0.8-8.6)$ & 0.12 & $\mathrm{NI}$ & $\mathrm{NI}$ \\
\hline $31-50$ & 145 & 43 & 29.7 & 102 & 70.3 & $2.4(0.8-7.4)$ & 0.12 & $\mathrm{NI}$ & $\mathrm{NI}$ \\
\hline $51-65$ & 175 & 58 & 33.1 & 117 & 66.9 & $2.9(0.9-8.6)$ & 0.06 & $\mathrm{NI}$ & $\mathrm{NI}$ \\
\hline$>65$ & 255 & 75 & 29.4 & 180 & 70.6 & $2.4(0.8-7.2)$ & 0.12 & $\mathrm{NI}$ & $\mathrm{NI}$ \\
\hline \multicolumn{7}{|l|}{$\mathrm{CCl}$} & 0.61 & $\mathrm{NA}$ & $\mathrm{NA}$ \\
\hline $0-1$ points & 428 & 130 & 30.4 & 298 & 69.6 & Ref & Ref & $\mathrm{NI}$ & $\mathrm{NI}$ \\
\hline 2-4 points & 217 & 60 & 27.6 & 157 & 72.4 & $0.9(0.6-1.3)$ & 0.47 & $\mathrm{NI}$ & $\mathrm{NI}$ \\
\hline$>4$ points & 53 & 18 & 34.0 & 35 & 66.0 & $1.2(0.6-2.2)$ & 0.59 & $\mathrm{NI}$ & $\mathrm{NI}$ \\
\hline \multicolumn{10}{|l|}{ Chronic alcohol abuse } \\
\hline Yes & 68 & 22 & 32.4 & 46 & 67.6 & $1.1(0.7-2.0)$ & 0.63 & $\mathrm{NI}$ & $\mathrm{NI}$ \\
\hline No & 630 & 186 & 29.5 & 444 & 70.5 & Ref & Ref & $\mathrm{NI}$ & $\mathrm{NI}$ \\
\hline \multicolumn{7}{|l|}{ Travel type } & 0.07 & Eliminated $^{\mathrm{b}}$ & Eliminated $^{b}$ \\
\hline Work/leisure/other & 553 & 154 & 27.8 & 399 & 72.2 & Ref & Ref & $\mathrm{NI}$ & $\mathrm{NI}$ \\
\hline Residence abroad & 96 & 34 & 35.4 & 62 & 64.6 & $1.4(0.9-2.2)$ & 0.13 & $\mathrm{NI}$ & $\mathrm{NI}$ \\
\hline VFR & 49 & 20 & 40.8 & 29 & 59.2 & $1.8(1.0-3.3)$ & 0.06 & $\mathrm{NI}$ & $\mathrm{NI}$ \\
\hline \multicolumn{7}{|l|}{ Geographical region } & $<0.01$ & NA & $<0.01$ \\
\hline North America & 3 & 1 & 33.3 & 2 & 66.7 & $2.1(0.2-23.9)$ & 0.54 & $1.4(0.1-16.0)$ & 0.79 \\
\hline Latin America, Caribbean & 10 & 6 & 60.0 & 4 & 40.0 & $6.4(1.8-23.3)$ & $<0.01$ & $6.4(1.7-23.7)$ & 0.01 \\
\hline Sub-Saharan Africa & 21 & 14 & 66.7 & 7 & 33.3 & $8.6(3.4-21.8)$ & $<0.01$ & $9.8(3.8-25.4)$ & $<0.01$ \\
\hline North Africa, Middle East & 38 & 19 & 50.0 & 19 & 50.0 & $4.3(2.2-8.4)$ & $<0.01$ & $4.1(2.1-8.2)$ & $<0.01$ \\
\hline Asia & 96 & 69 & 71.9 & 27 & 28.1 & $\begin{array}{c}11.0 \\
(6.7-18.0) \\
\end{array}$ & $<0.01$ & $10.5(6.3-17.3)$ & $<0.01$ \\
\hline Oceania & 6 & 0 & 0 & 6 & 100 & NA & NA & NA & $\mathrm{NA}$ \\
\hline Europe & 524 & 99 & 18.9 & 425 & 81.1 & Ref & Ref & Ref & Ref \\
\hline \multicolumn{7}{|l|}{ Duration of hospitalisation } & $<0.01$ & Eliminated $^{\mathrm{b}}$ & Eliminated $^{\mathrm{b}}$ \\
\hline $1-2$ days & 83 & 14 & 16.9 & 69 & 83.1 & Ref & Ref & $\mathrm{NI}$ & $\mathrm{NI}$ \\
\hline 3-7 days & 208 & 48 & 23.1 & 160 & 76.9 & $1.5(0.8-2.9)$ & 0.25 & $\mathrm{NI}$ & $\mathrm{NI}$ \\
\hline $8-14$ days & 186 & 56 & 30.1 & 130 & 69.9 & $2.1(1.1-4.1)$ & 0.02 & $\mathrm{NI}$ & $\mathrm{NI}$ \\
\hline Over 14 days & 193 & 77 & 39.9 & 116 & 60.1 & $3.3(1.7-6.2)$ & $<0.01$ & $\mathrm{NI}$ & $\mathrm{NI}$ \\
\hline Data missing & 28 & 13 & 46.4 & 15 & 53.6 & $\mathrm{NI}$ & $\mathrm{NI}$ & $\mathrm{NI}$ & $\mathrm{NI}$ \\
\hline \multicolumn{10}{|l|}{ ICU treatment abroad } \\
\hline Yes & 190 & 76 & 40.0 & 114 & 60.0 & $1.9(1.3-2.7)$ & $<0.01$ & $1.8(1.2-2.7)$ & 0.01 \\
\hline No & 508 & 132 & 26.0 & 376 & 74.0 & Ref & Ref & Ref & Ref \\
\hline \multicolumn{8}{|c|}{ Major invasive procedure abroad } & Eliminated $^{\mathrm{b}}$ & Eliminated $^{\mathrm{b}}$ \\
\hline Yes & 290 & 102 & 35.2 & 188 & 64.8 & $1.5(1.1-2.1)$ & 0.01 & $\mathrm{NI}$ & $\mathrm{NI}$ \\
\hline No & 408 & 106 & 26.0 & 302 & 74.0 & Ref & Ref & $\mathrm{NI}$ & $\mathrm{NI}$ \\
\hline \multicolumn{10}{|l|}{ Antibiotic use abroad } \\
\hline Yes & 383 & 141 & 36.8 & 242 & 63.2 & $2.2(1.5-3.0)$ & $<0.01$ & $1.6(1.1-2.4)$ & 0.02 \\
\hline No & 315 & 67 & 21.3 & 248 & 78.7 & Ref & Ref & Ref & Ref \\
\hline \multicolumn{10}{|l|}{ Reason for hospitalisation } \\
\hline Trauma & 323 & 96 & 29.7 & 227 & 70.3 & Ref & Ref & $\mathrm{NI}$ & $\mathrm{NI}$ \\
\hline Non-trauma & 375 & 112 & 29.9 & 263 & 70.1 & $1.0(0.7-1.4)$ & 0.97 & $\mathrm{NI}$ & $\mathrm{NI}$ \\
\hline Antibiotic use during screer & & & & & & & & & \\
\hline Yes & 182 & 69 & 37.9 & 113 & 62.1 & $1.8(1.2-2.6)$ & $<0.01$ & $\mathrm{NI}$ & $\mathrm{NI}$ \\
\hline No & 458 & 117 & 25.5 & 341 & 74.5 & Ref & Ref & $\mathrm{NI}$ & $\mathrm{NI}$ \\
\hline Data missing & 58 & 22 & 37.9 & 36 & 62.1 & $\mathrm{NI}$ & $\mathrm{NI}$ & $\mathrm{NI}$ & $\mathrm{NI}$ \\
\hline
\end{tabular}

AOR: adjusted odds ratio; CCI: Charlson Comorbidity Index; CI: confidence interval; ICU: intensive care unit; NA: not applicable; NI: not included; OR: odds ratio;

a The following variables were included in multivariable analysis: travel type, geographical region, duration of hospitalisation, ICU treatment, major invasive procedure, antibiotic use abroad.

${ }^{\mathrm{b}}$ Eliminated before final step in backward selection. 
Culture-based analyses identified 261 ESBL-PE, 25 MRSA, 26 VRE, 14 MDRPA, 24 CPE and 33 MDRACI strains, 30 of which were confirmed as Acinetobacter baumannii and three as non-specified Acinetobacter species. Among ESBL-PE carriers, for 102 patients (62.6\%) one ESBL-PE strain had been recorded, for 33 (20.2\%) two, and for $28(17.2 \%)$ three or more. A total of 46 carbapenemase gene-positive (CPE, MDRACI or MDRPA) strains had been recorded for 33 patients, seven carbapenemase gene-negative MDRPA strains for six and one gene-negative MDRACI strain for one. From 2010 to 2012, carbapenemase genes were not tested in five MDRPA strains carried by four patients, nor in four MDRACl strains, each from one patient. The carbapenemase gene-negative and non-tested strains were included in the figures for colonised patients.

\section{Risk factors for colonisation with multidrug- resistant bacteria}

In univariate analysis, several factors were associated with MDR carriage (Table 1) and in multivariable analysis, geographical region, ICU treatment and antibiotic use abroad were identified as independent risk factors. Separate analyses of individual MDR types are presented below.

Univariate analysis indicated that country of hospitalisation, antibiotic use abroad, duration of hospitalisation and surgery were risk factors associated with ESBL-PE carriage (Table 2), whereas multivariable analysis yielded only geographical region as risk factor.

Results of the univariate analyses conducted for MDRACI and MRSA are presented in Tables 3 and 4. Carriage of each of these two was associated with antibiotic use abroad, while only MDRACl carriage was associated also with antibiotics during screening. In addition, both were associated with ICU treatment abroad. Colonisation with $\mathrm{MDRACl}$ was more common among those hospitalised in Asia (14/96; 14.6\%) than Europe $(10 / 524 ; 1.9 \%)$. Carriage of MRSA was associated with chronic alcohol abuse, a finding independent of antibiotic use in a model with two explanatory variables. Significant associations were also recorded between $\mathrm{CCl}$ and MDRACI (Table 3) and between duration of hospitalisation and MRSA (Table 4).

For VRE, the following factors were associated with colonisation: antibiotic use abroad (odds ratio $(O R)=3.4$; 95\% confidence interval $(\mathrm{Cl}): 1.3-9.2 ; \mathrm{p}=0.01)$, antibiotic use during VRE screening $(\mathrm{OR}=2.3 ; 95 \% \mathrm{Cl}$ : 1.0-5.3; $p=0.048), I C U$ treatment abroad (OR = 3.0; 95\% Cl: 1.3-6.7; $\mathrm{p}=0.005$ ), and travel type (travellers VFR vs work/holiday/other OR $=5.2 ; 95 \% \mathrm{Cl}: 1.9-14.3$; $p=0.001$ ). As for other factors in Tables 3 and 4, no significant association was found (data not shown).

Risk factor analyses were not carried out for CPE or MDRPA due to the small number of colonised individuals ( 14 with CPE and 12 with MDRPA).

\section{Clinical infections caused by multidrug-} resistant bacteria

During post-transfer hospitalisation, 22 of 698 patients (3.2\% of the whole study population and $10.6 \%$ of those identified as MDR bacteria carriers) had a microbiologically verified clinical MDR bacterial infection, most commonly pneumonia which was found in nine patients $(1.3 \%$ of all), surgical site infection (six patients, $0.9 \%$ ), and urinary tract infection (six patients, 0.9\%). One patient had MDR bacteraemia. For sites of infection and causative MDR bacteria, see Supplement (Supplementary Table S4). Infection by MDR bacteria was recorded as cause of death for two patients (0.3\%).

\section{Discussion}

Of the 698 patients transferred to a Finnish hospital directly from hospitals abroad, $29.8 \%$ were colonised by MDR bacteria, the rates varying considerably by geographical region visited. From these 208 patients, 383 MDR bacterial strains were recorded. While these figures indicate the burden of MDR bacteria related to interhospital transfer, closer scrutiny reveals background data applicable to infection control practices and even choice of empiric antibiotics.

At first glance, the $29.8 \%$ overall MDR carriage rate appears low. As the rates typically decrease after patients return to low-prevalence countries [18-23], one could expect colonisation to be particularly common in these patients who were screened soon after return to Finland. By contrast, the prevalence proved to be similar to that of our previous data from 2010 to 2013 showing $29.7 \%$ carriage rates for patients screened within 12 months after hospitalisation abroad [13]. However, the closeness of the rates may be ascribed to at least two factors. Firstly, up to $23 \%$ of the patients in that previous investigation were, in fact, direct-transfer patients, and the time taken from return from abroad to sampling for the rest of the patients was short (median: 11 days). Secondly, the proportion of patients hospitalised in Europe where acquisition is less common $[13,15]$ was higher in the current $(75 \%)$ than in the previous dataset $(64 \%)$.

Other European studies have reported MDR bacterial colonisation in similar patient groups but with differences in research design [5-9,11,12]. Among 1,167 patients directly transferred from hospitals abroad to the Netherlands between 1998 and 2001, Kaiser et al. show a colonisation rate of $18.2 \%$ [6], but their designation of resistant Gram-negative bacteria was solely based on gentamicin resistance [6] and there has been a substantial general increase in AMR rates since that study. In more recent research among patients screened on direct transfer or within 14 days of hospitalisation abroad, between 7.2 and $28.6 \%$ were colonised $[7-9,11,12]$. For patients hospitalised abroad and examined within 14 days in Switzerland between 2009 and 2011, Nemeth et al. showed the rate to be $17 \%$, although VRE was not included in this study [7]. 
TABLE 2

Patient characteristics and risk factor analyses of ESBL-producing Enterobacteriaceae colonisation among patients screened after direct transfer from hospitals abroad to Helsinki University Hospital, Finland, 2010-2019 (n=698)

\begin{tabular}{|c|c|c|c|c|c|c|c|c|c|}
\hline & \multirow{2}{*}{$\begin{array}{c}\text { Patients } \\
(n=698)\end{array}$} & \multicolumn{2}{|c|}{$\begin{array}{l}\text { ESBL-PE-positive } \\
\quad(n=163)\end{array}$} & \multicolumn{2}{|c|}{$\begin{array}{l}\text { ESBL-PE-negative } \\
\quad(n=535)\end{array}$} & \multirow{2}{*}{$\begin{array}{l}\text { OR }(95 \% \mathrm{Cl}) \\
\text { in univariate } \\
\text { analysis }\end{array}$} & \multirow{2}{*}{$\begin{array}{l}\mathrm{p} \text { value in } \\
\text { univariate } \\
\text { analysis }\end{array}$} & \multirow{2}{*}{$\begin{array}{c}\text { AOR }(95 \% \mathrm{Cl}) \\
\text { in multivariable } \\
\text { analysis }^{\mathrm{a}}\end{array}$} & \multirow{2}{*}{$\begin{array}{l}\mathrm{p} \text { value in } \\
\text { multivariable } \\
\text { analysis }^{\mathrm{a}}\end{array}$} \\
\hline & & $\mathrm{n}$ & $\%$ & $n$ & $\%$ & & & & \\
\hline \multicolumn{10}{|l|}{ Sex } \\
\hline Male & 427 & 105 & 24.6 & 322 & 75.4 & $1.2(0.8-1.7)$ & 0.33 & $\mathrm{NI}$ & $\mathrm{NI}$ \\
\hline Female & 271 & 58 & 21.4 & 213 & 78.6 & Ref & Ref & $\mathrm{NI}$ & $\mathrm{NI}$ \\
\hline \multicolumn{7}{|l|}{ Age group (years) } & 0.39 & \multicolumn{2}{|c|}{ NA } \\
\hline $0-5$ & 27 & 4 & 14.8 & 23 & 85.2 & Ref & Ref & $\mathrm{NI}$ & $\mathrm{NI}$ \\
\hline $6-17$ & 35 & 9 & 25.7 & 26 & 74.3 & $2.0(0.5-7.3)$ & 0.30 & $\mathrm{NI}$ & $\mathrm{NI}$ \\
\hline $18-30$ & 61 & 16 & 26.2 & 45 & 73.8 & $2.0(0.6-6.8)$ & 0.25 & $\mathrm{NI}$ & $\mathrm{NI}$ \\
\hline $31-50$ & 145 & 36 & 24.8 & 109 & 75.2 & $1.9(0.6-5.9)$ & 0.27 & $\mathrm{NI}$ & $\mathrm{NI}$ \\
\hline $51-65$ & 175 & 48 & 27.4 & 127 & 72.6 & $2.2(0.7-6.6)$ & 0.17 & $\mathrm{NI}$ & $\mathrm{NI}$ \\
\hline$>65$ & 255 & 50 & 19.6 & 205 & 80.4 & $1.4(0.5-4.2)$ & 0.55 & $\mathrm{NI}$ & $\mathrm{NI}$ \\
\hline \multicolumn{7}{|l|}{$\mathrm{CCl}$} & 0.64 & \multicolumn{2}{|c|}{ NA } \\
\hline o-1 points & 428 & 105 & 24.5 & 323 & 75.5 & Ref & Ref & $\mathrm{NI}$ & $\mathrm{NI}$ \\
\hline 2-4 points & 217 & 47 & 21.7 & 170 & 78.3 & $0.9(0.6-1.3)$ & 0.42 & $\mathrm{NI}$ & $\mathrm{NI}$ \\
\hline$>4$ points & 53 & 11 & 20.8 & 42 & 79.2 & $0.8(0.4-1.6)$ & 0.55 & $\mathrm{NI}$ & $\mathrm{N}$ \\
\hline \multicolumn{10}{|l|}{ Chronic alcohol abuse } \\
\hline Yes & 68 & 14 & 20.6 & 54 & 79.4 & Ref & Ref & $\mathrm{NI}$ & $\mathrm{NI}$ \\
\hline No & 630 & 149 & 23.7 & 481 & 76.3 & $1.2(0.6-2.2)$ & 0.57 & $\mathrm{NI}$ & $\mathrm{NI}$ \\
\hline \multicolumn{7}{|l|}{ Travel type } & 0.26 & \multicolumn{2}{|c|}{ NA } \\
\hline Work/leisure/other & 553 & 122 & 22.1 & 431 & 77.9 & Ref & Ref & $\mathrm{NI}$ & $\mathrm{NI}$ \\
\hline Residence abroad & 96 & 26 & 27.1 & 70 & 72.9 & $1.3(0.8-2.1)$ & 0.28 & $\mathrm{NI}$ & $\mathrm{NI}$ \\
\hline VFR & 49 & 15 & 30.6 & 34 & 69.4 & $1.6(0.8-3.0)$ & 0.17 & $\mathrm{NI}$ & $\mathrm{NI}$ \\
\hline \multicolumn{7}{|l|}{ Geographical region } & $<0.01$ & NA & $<0.01$ \\
\hline North America & 3 & o & 0 & 3 & 100 & NA & NA & NA & NA \\
\hline Latin America, Caribbean & 10 & 6 & 60.0 & 4 & 40.0 & $9.7(2.7-35.3)$ & $<0.01$ & $9.7(2.7-35.4)$ & $<0.01$ \\
\hline Sub-Saharan Africa & 21 & 13 & 61.9 & 8 & 38.1 & $10.5(4.2-26.3)$ & $<0.01$ & $13.1(4.7-37.0)$ & $<0.01$ \\
\hline North Africa, Middle East & 38 & 13 & 34.2 & 25 & 65.8 & $3.4(1.6-6.9)$ & $<0.01$ & $3.7(1.8-7.6)$ & $<0.01$ \\
\hline Asia & 96 & 61 & 63.5 & 35 & 36.5 & $11.3(7.0-18.4)$ & $<0.01$ & $10.4(6.3-17.2)$ & $<0.01$ \\
\hline Oceania & 6 & 0 & 0 & 6 & 100 & NA & NA & NA & NA \\
\hline Europe & 524 & 70 & 13.4 & 454 & 86.6 & Ref & Ref & Ref & Ref \\
\hline \multicolumn{7}{|l|}{ Duration of hospitalisation } & 0.03 & Eliminated $^{b}$ & Eliminated $^{b}$ \\
\hline $1-2$ days & 83 & 12 & 14.5 & 71 & 85.5 & Ref & Ref & $\mathrm{NI}$ & $\mathrm{NI}$ \\
\hline 3-7 days & 208 & 39 & 18.8 & 169 & 81.3 & $1.4(0.7-2.8)$ & 0.39 & $\mathrm{NI}$ & $\mathrm{NI}$ \\
\hline $8-14$ days & 186 & 46 & 24.7 & 140 & 75.3 & $1.9(1.0-3.9)$ & 0.06 & $\mathrm{NI}$ & $\mathrm{NI}$ \\
\hline Over 14 days & 193 & 55 & 28.5 & 138 & 71.5 & $2.4(1.2-4.7)$ & 0.01 & $\mathrm{NI}$ & $\mathrm{NI}$ \\
\hline Data missing & 28 & 11 & 39.3 & 17 & 60.7 & $\mathrm{NI}$ & $\mathrm{NI}$ & $\mathrm{NI}$ & $\mathrm{NI}$ \\
\hline \multicolumn{10}{|l|}{ ICU treatment abroad } \\
\hline Yes & 190 & 50 & 26.3 & 140 & 73.7 & $1.2(0.9-1.8)$ & 0.26 & $\mathrm{NI}$ & $\mathrm{NI}$ \\
\hline No & 508 & 113 & 22.2 & 395 & 77.8 & Ref & Ref & $\mathrm{NI}$ & $\mathrm{NI}$ \\
\hline Major invasive procedure & road & & & & & & & & \\
\hline Yes & 290 & 79 & 27.2 & 211 & 72.8 & $1.4(1.0-2.1)$ & 0.04 & $1.5(1.0-2.2)$ & 0.07 \\
\hline No & 408 & 84 & 20.6 & 324 & 79.4 & Ref & Ref & Ref & Ref \\
\hline Antibiotic use abroad & & & & & & & & Eliminated $^{b}$ & Eliminated $^{b}$ \\
\hline Yes & 383 & 104 & 27.2 & 279 & 72.8 & $1.6(1.1-2.3)$ & 0.01 & $\mathrm{NI}$ & $\mathrm{NI}$ \\
\hline No & 315 & 59 & 18.7 & 256 & 81.3 & Ref & Ref & $\mathrm{NI}$ & $\mathrm{NI}$ \\
\hline Reason for hospitalisation & & & & & & & & & \\
\hline Trauma & 323 & 79 & 24.5 & 244 & 75.5 & $1.1(0.8-1.6)$ & 0.52 & $\mathrm{NI}$ & $\mathrm{NI}$ \\
\hline Non-trauma & 375 & 84 & 22.4 & 291 & 77.6 & Ref & Ref & $\mathrm{NI}$ & $\mathrm{NI}$ \\
\hline Antibiotic use during scre & ing $^{\mathrm{c}}$ & & & & & & & & \\
\hline Yes & 199 & 51 & 25.6 & 148 & 74.4 & $1.3(0.9-1.9)$ & 0.23 & $\mathrm{NI}$ & $\mathrm{NI}$ \\
\hline No & 472 & 101 & 21.4 & 371 & 78.6 & Ref & Ref & $\mathrm{NI}$ & $\mathrm{NI}$ \\
\hline Data missing & 27 & 11 & 40.7 & 16 & 59.3 & $\mathrm{NI}$ & $\mathrm{NI}$ & $\mathrm{NI}$ & $\mathrm{NI}$ \\
\hline
\end{tabular}

AOR: adjusted odds ratio; CCI: Charlson Comorbidity Index; CI: confidence interval; ICU: intensive care unit; ESBL-PE: extended-spectrum $\beta$-lactamase-producing Enterobacteriaceae; NA: not applicable; NI: not included; OR: odds ratio; Ref: reference; VFR: visiting friends and relatives.

a The following variables were included in multivariable analysis: geographical region, duration of hospitalisation, major invasive procedure, antibiotic use abroad.

${ }^{b}$ Eliminated before final step in backward selection.

Antibiotic treatment during screening for multidrug-resistant Gram-negative bacteria (MDRGNB). 
TABLE 3

Patient characteristics and risk factor analyses of multidrug-resistant Acinetobacter spp. colonisation among patients screened after direct transfer from hospitals abroad to Helsinki University Hospital, Finland, 2010-2019 (n=698)

\begin{tabular}{|c|c|c|c|c|c|c|c|}
\hline & \multirow{2}{*}{$\begin{array}{c}\text { Patients }(n=698) \\
n\end{array}$} & \multicolumn{2}{|c|}{$\begin{array}{l}\text { MDRACI-positive } \\
\quad(\mathrm{n}=28)\end{array}$} & \multicolumn{2}{|c|}{$\begin{array}{l}\text { MDRACI-negative } \\
\quad(n=670)\end{array}$} & \multirow{2}{*}{$\begin{array}{l}\text { OR }(95 \% \mathrm{Cl}) \text { in } \\
\text { univariate analysis }\end{array}$} & \multirow{2}{*}{$\begin{array}{l}\text { p value in univariate } \\
\text { analysis }\end{array}$} \\
\hline & & $\mathrm{n}$ & $\%$ & $n$ & $\%$ & & \\
\hline \multicolumn{8}{|l|}{ Sex } \\
\hline Male & 427 & 21 & 4.9 & 406 & 95.1 & $2.0(0.8-4.7)$ & 0.13 \\
\hline Female & 271 & 7 & 2.6 & 264 & 97.4 & Ref & Ref \\
\hline \multicolumn{7}{|l|}{ Age group (years) } & 0.34 \\
\hline $0-5$ & 27 & 0 & 0 & 27 & 100 & NA & NA \\
\hline $6-17$ & 35 & 1 & 2.9 & 34 & 97.1 & $0.5(0.1-3.7)$ & 0.47 \\
\hline $18-30$ & 61 & 2 & 3.3 & 59 & 96.7 & $0.5(0.1-2.4)$ & 0.43 \\
\hline $31-50$ & 145 & 8 & 5.5 & 137 & 94.5 & $0.9(0.4-2.3)$ & 0.88 \\
\hline $51-65$ & 175 & 2 & 1.1 & 173 & 98.9 & $0.2(0.0-0.8)$ & 0.03 \\
\hline$>65$ & 255 & 15 & 5.9 & 240 & 94.1 & 1.0 & Ref \\
\hline \multicolumn{7}{|l|}{$\mathrm{CCl}$} & 0.02 \\
\hline o-1 points & 428 & 10 & 2.3 & 418 & 97.7 & Ref & Ref \\
\hline 2-4 points & 217 & 14 & 6.5 & 203 & 93.5 & $2.9(1.3-6.6)$ & 0.01 \\
\hline$>4$ points & 53 & 4 & 7.5 & 49 & 92.5 & $3.4(1.0-11.3)$ & 0.04 \\
\hline \multicolumn{8}{|l|}{ Chronic alcohol abuse } \\
\hline Yes & 68 & 3 & 4.4 & 65 & 95.6 & $1.1(0.3-3.8)$ & 0.75 \\
\hline No & 630 & 25 & 4.0 & 605 & 96.0 & Ref & Ref \\
\hline \multicolumn{7}{|l|}{ Travel type } & 0.22 \\
\hline Work/leisure/other & 553 & 19 & 3.4 & 534 & 96.6 & Ref & Ref \\
\hline Residence abroad & 96 & 7 & 7.3 & 89 & 92.7 & $2.2(0.9-5.4)$ & 0.08 \\
\hline VFR & 49 & 2 & 4.1 & 47 & 95.9 & $1.2(0.3-5.3)$ & 0.81 \\
\hline \multicolumn{7}{|l|}{ Geographical region } & $<0.01$ \\
\hline North America & 3 & 0 & 0 & 3 & 100 & NA & NA \\
\hline Latin America, Caribbean & 10 & 1 & 10.0 & 9 & 90.0 & $5.7(0.7-49.5)$ & 0.11 \\
\hline Sub-Saharan Africa & 21 & o & 0 & 21 & 100 & NA & NA \\
\hline North Africa, Middle East & 38 & 3 & 7.9 & 35 & 92.1 & $4.4(1.2-16.7)$ & 0.03 \\
\hline Asia & 96 & 14 & 14.6 & 82 & 85.4 & $8.8(3.8-20.4)$ & $<0.01$ \\
\hline Oceania & 6 & 0 & 0 & 6 & 100 & NA & NA \\
\hline Europe & 524 & 10 & 1.9 & 514 & 98.1 & Ref & Ref \\
\hline \multicolumn{7}{|l|}{ Duration of hospitalisation } & 0.14 \\
\hline $1-2$ days & 83 & 1 & 1.2 & 82 & 98.8 & Ref & Ref \\
\hline 3-7 days & 208 & 6 & 2.9 & 202 & 97.1 & $2.4(0.3-20.5)$ & 0.41 \\
\hline $8-14$ days & 186 & 7 & 3.8 & 179 & 96.2 & $3.2(0.4-26.5)$ & 0.28 \\
\hline Over 14 days & 193 & 13 & 6.7 & 180 & 93.3 & $5.9(0.8-46.0)$ & 0.09 \\
\hline Data missing & 28 & 1 & 3.6 & 27 & 96.4 & $\mathrm{NI}$ & $\mathrm{NI}$ \\
\hline \multicolumn{8}{|l|}{ ICU treatment abroad } \\
\hline Yes & 190 & 20 & 10.5 & 170 & 89.5 & $7.4(3.2-17.0)$ & $<0.01$ \\
\hline No & 508 & 8 & 1.6 & 500 & 98.4 & Ref & Ref \\
\hline \multicolumn{8}{|c|}{ Major invasive procedure abroad } \\
\hline Yes & 290 & 16 & 5.5 & 274 & 94.5 & $1.9(0.9-4.1)$ & 0.09 \\
\hline No & 408 & 12 & 2.9 & 396 & 97.1 & Ref & Ref \\
\hline \multicolumn{8}{|l|}{ Antibiotic use abroad } \\
\hline Yes & 383 & 27 & 7.0 & 356 & 93.0 & $23.8(3.2-176.3)$ & $<0.01$ \\
\hline No & 315 & 1 & 0.3 & 314 & 99.7 & Ref & Ref \\
\hline \multicolumn{8}{|l|}{ Reason for hospitalisation } \\
\hline Trauma & 323 & 12 & 3.7 & 311 & 96.3 & Ref & Ref \\
\hline Non-trauma & 375 & 16 & 4.3 & 359 & 95.7 & $1.2(0.5-2.5)$ & 0.71 \\
\hline \multicolumn{8}{|c|}{ Antibiotic use during screening ${ }^{a}$} \\
\hline Yes & 199 & 13 & 6.5 & 186 & 93.5 & $2.9(1.3-6.7)$ & $<0.01$ \\
\hline No & 472 & 11 & 2.3 & 461 & 97.7 & Ref & Ref \\
\hline Data missing & 27 & 4 & 14.8 & 23 & 85.2 & $\mathrm{NI}$ & $\mathrm{NI}$ \\
\hline
\end{tabular}

AOR: adjusted odds ratio; CCl: Charlson Comorbidity Index; Cl: confidence interval; ICU: intensive care unit; NA: not applicable; NI: not included; OR: odds ratio; Ref: reference; VFR: visiting friends and relatives.

a Antibiotic treatment during screening for multidrug-resistant Gram-negative bacteria (MDRGNB). 
In another Swiss study where outpatient treatment abroad was also included but without screening for VRE, Kaspar et al. report a $16.3 \%$ carriage rate for direct transfer patients in 2012 to 2013 [11]. Josseaume et al. (2010-2011) and Birgand et al. (2012-2013) show MDR bacterial colonisation for $7.2 \%$ and $28.6 \%$ of repatriated patients in France, respectively [8,9]. In 2012 to 2013, Mutters et al. detected a colonisation rate of 21.0\% among patients hospitalised abroad at least 48 $\mathrm{h}$ and subsequently transferred to a German hospital [12].

The present study identified region visited, ICU treatment, and antibiotic use during travel as independent risk factors of MDR bacterial colonisation. All three factors have previously been associated with MDR acquisition [13,24-27]. In our study population, association with geographical region proved particularly strong, for example, the colonisation rate was $18.9 \%$ among patients transferred from European countries and $71.9 \%$ among those returned from Asia ( $p<0.001$; $\mathrm{OR}=10.5 ; 95 \% \mathrm{Cl}: 6.3-17.3)$. This difference was mainly ascribed to Gram-negative MDR bacteria rather than MRSA or VRE.

The overall colonisation rate by ESBL-PE was $23.4 \%$, accounting for $68.1 \%$ of all MDR strains identified. This rate is substantially higher than among the general Finnish population: in 2009 to $2010,1.2 \%$ of 430 Finnish travellers were colonised with faecal ESBL-PE before travel [27]; in 2015 to 2017, a study among Finnish elective surgery patients and medical students reported that $4.7 \%$ were colonised with ESBL-producing $E$. coli, and $1.1 \%$ with ESBL-producing $K$. pneumoniae [28].

Several prospective investigations have shown that visitors to (sub)tropical regions acquire ESBL-PE, with antibiotic use predisposing to colonisation $[18,27,29]$. In the present study looking at hospitalised travellers, multivariable analysis identified region of hospitalisation as the sole factor independently associated with ESBL-PE colonisation. ESBL-PE acquisition is so common among travellers visiting high-risk regions that any additional impact of nosocomial transmission may remain modest. While antibiotic use was found to predispose to MDR colonisation as a whole, its association with contracting ESBL-PE was significant in univariate but not in multivariable analysis, possibly because of an insufficient number of observations.

The colonisation rates for other MDR bacteria were low, but not without relevance in a low-prevalence country like Finland [30]. In the stool specimens of 33 patients within this study, 46 carbapenemase-producing strains were recorded: $20 \mathrm{CPE}$ and $26 \mathrm{MDRACl}$ or MDRPA. In comparison, between 2010 and 2018, only 136 CPE strains were reported for the entire Helsinki and Uusimaa hospital district serving a population of 1.7 million, and the rates of MDRACI and MDRPA have been very low $[30,31]$. Thus, direct hospital transfers evidently contribute considerably to these cases. The
MRSA colonisation rate of $3.6 \%$ is in line with that of $1.2-4.1 \%$ observed in earlier studies $[5-8,12]$. Although the rates for MRSA (3.6\%) and VRE (3.8\%) may appear low, they exceed those typical for Finland in general [30].

In univariate analyses conducted separately for MDRACI, MRSA and VRE, we found several associations. Antibiotic use abroad and ICU treatment were associated with each of the three. Indeed, both antibiotic use and intensive care predispose to MDR acquisition [24-26]. The association observed between MRSA carriage and chronic alcohol abuse confirms results of a previous study [32].

Clinical, microbiologically verified MDR bacterial infections were identified in $10.6 \%$ of the colonised patients in our study within 30 days after transfer, consistent with the rate of $11.4 \%$ observed in 2010 to 2013 as reported by Khawaja et al. [13] The rate of infection observed in the study by Mutters et al. was significantly higher at $29.9 \%$, however, the demographics of patients in that study were different: over half of the patients were transferred to Germany from their country of origin in the Middle East [12]. As for nonhospitalised healthy travellers, we recently showed a maximum clinical infection rate of $17 \%$ for ESBL-PE carriers, with travellers' diarrhoea (TD) as the most common manifestation, whereas the estimated maximal rate of infections other than TD was 3\% [4]. Indeed, the data suggested higher rates of clinical MDR infections (TD excluded) among travellers who were hospitalised compared with non-hospitalised travellers [4].

\section{Limitations of the study}

This investigation had limitations typical of a retrospective study design, such as incomplete data in some patient records and missing information on pretravel colonisation. Comparisons can nevertheless be made with background colonisation rates, as mentioned above. Due to lack of non-hospitalised controls, it is difficult to determine the respective proportions of nosocomial and community-acquired infection during travel. In numerous reports on ESBL-PE colonisation after travel without hospitalisation [1] the rates resemble our data, but community acquisition of other MDR bacteria appears limited [27,29,33-35]. Furthermore, since the time frame for recording MDR bacteria was up to 1 month, nosocomial transmission after return to Finland cannot be ruled out. However, the risk was considered marginal on account of the low background prevalence: only $2 \%$ of $S$. aureus isolates are MRSA strains [30], and the background colonisation rate of ESBL-PE in Finland remains under 5\% [28].

As culture-based assays lack sensitivity, a greater number of different MDR bacterial strains could be expected when employing modern genome-based methods, as shown in our recent study on travellers [36]. However, culture-based approaches are used in clinical practice and allow comparisons with earlier studies. For some 
TABLE 4

Patient characteristics and risk factor analyses of meticillin-resistant Staphylococcus aureus colonisation among patients screened after transfer directly from hospitals abroad to Helsinki University Hospital, Finland, 2010-2019 (n=698)

\begin{tabular}{|c|c|c|c|c|c|c|c|c|c|}
\hline & \multirow{2}{*}{$\begin{array}{c}\text { Patients } \\
(n=698) \\
n\end{array}$} & \multicolumn{2}{|c|}{$\begin{array}{l}\text { MRSA positive } \\
\quad(n=25)\end{array}$} & \multicolumn{2}{|c|}{$\begin{array}{l}\text { MRSA negative } \\
\quad(n=673)\end{array}$} & \multirow{2}{*}{$\begin{array}{c}\text { OR }(95 \% \mathrm{Cl}) \\
\text { in univariate } \\
\text { analysis }\end{array}$} & \multirow{2}{*}{$\begin{array}{l}\mathrm{p} \text { value in } \\
\text { univariate } \\
\text { analysis }\end{array}$} & \multirow{2}{*}{$\begin{array}{c}\text { AOR }(95 \% \mathrm{Cl}) \\
\text { in multivariable } \\
\text { analysis }^{\mathrm{a}}\end{array}$} & \multirow{2}{*}{$\begin{array}{l}\text { p value in } \\
\text { multivariable } \\
\text { analysis }^{\mathrm{a}}\end{array}$} \\
\hline & & $\mathrm{n}$ & $\%$ & $n$ & $\%$ & & & & \\
\hline \multicolumn{10}{|l|}{ Sex } \\
\hline Male & 427 & 17 & 4.0 & 410 & 96.0 & $1.4(0.6-3.2)$ & 0.48 & $\mathrm{NI}$ & $\mathrm{NI}$ \\
\hline Female & 271 & 8 & 3.0 & 263 & 97.0 & Ref & Ref & $\mathrm{NI}$ & $\mathrm{NI}$ \\
\hline \multicolumn{7}{|l|}{ Age group (years) } & 0.69 & NA & \\
\hline $0-5$ & 27 & 0 & o & 27 & 100 & NA & NA & $\mathrm{NI}$ & $\mathrm{NI}$ \\
\hline $6-17$ & 35 & 1 & 2.9 & 34 & 97.1 & $0.5(0.1-4.3)$ & 0.57 & $\mathrm{NI}$ & $\mathrm{NI}$ \\
\hline $18-30$ & 61 & 1 & 1.6 & 60 & 98.4 & $0.3(0.0-2.4)$ & 0.26 & $\mathrm{NI}$ & $\mathrm{NI}$ \\
\hline $31-50$ & 145 & 3 & 2.1 & 142 & 97.9 & $0.4(0.1-1.4)$ & 0.15 & $\mathrm{NI}$ & $\mathrm{NI}$ \\
\hline $51-65$ & 175 & 7 & 4.0 & 168 & 96.0 & $0.8(0.3-2.0)$ & 0.60 & $\mathrm{NI}$ & $\mathrm{NI}$ \\
\hline$>65$ & 255 & 13 & 5.1 & 242 & 94.9 & Ref & Ref & $\mathrm{NI}$ & $\mathrm{NI}$ \\
\hline \multicolumn{7}{|l|}{$\mathrm{CCl}$} & 0.36 & NA & \\
\hline o-1 points & 428 & 12 & 2.8 & 416 & 97.2 & Ref & $\begin{array}{r}\text { Ref } \\
\end{array}$ & $\mathrm{NI}$ & $\mathrm{NI}$ \\
\hline 2-4 points & 217 & 10 & 4.6 & 207 & 95.4 & $1.7(0.7-3.9)$ & 0.24 & $\mathrm{NI}$ & $\mathrm{NI}$ \\
\hline$>4$ points & 53 & 3 & 5.7 & 50 & 94.3 & $2.1(0.6-7.6)$ & 0.27 & $\mathrm{NI}$ & $\mathrm{NI}$ \\
\hline \multicolumn{10}{|l|}{ Chronic alcohol abuse } \\
\hline Yes & 68 & 6 & 8.8 & 62 & 91.2 & $3.1(1.2-8.1)$ & 0.03 & $2.9(1.1-7.5)$ & 0.03 \\
\hline No & 630 & 19 & 3.0 & 611 & 97.0 & Ref & Ref & Ref & Ref \\
\hline \multicolumn{7}{|l|}{ Travel type } & 0.56 & NA & \\
\hline Work/leisure/other & 553 & 18 & 3.3 & 535 & 96.7 & Ref & Ref & $\mathrm{NI}$ & $\mathrm{NI}$ \\
\hline Residence abroad & 96 & 4 & 4.2 & 92 & 95.8 & $1.3(0.4-3.9)$ & 0.65 & $\mathrm{NI}$ & $\mathrm{NI}$ \\
\hline VFR & 49 & 3 & 6.1 & 46 & 93.9 & $1.9(0.6-6.8)$ & 0.30 & $\mathrm{NI}$ & $\mathrm{NI}$ \\
\hline \multicolumn{7}{|l|}{ Geographical region } & 0.14 & NA & \\
\hline North America & 3 & o & 0 & 3 & 100 & NA & NA & $\mathrm{NI}$ & $\mathrm{NI}$ \\
\hline Latin America, Caribbean & 10 & 1 & 10.0 & 9 & 90.0 & $4.4(0.5-37.1)$ & 0.18 & $\mathrm{NI}$ & $\mathrm{NI}$ \\
\hline Sub-Saharan Africa & 21 & 2 & 9.5 & 19 & 90.5 & $4.1(0.9-19.6)$ & 0.07 & $\mathrm{NI}$ & $\mathrm{NI}$ \\
\hline North Africa, Middle East & 38 & 4 & 10.5 & 34 & 89.5 & $4.6(1.4-14.9)$ & 0.01 & $\mathrm{NI}$ & $\mathrm{NI}$ \\
\hline Asia & 96 & 5 & 5.2 & 91 & 94.8 & $2.2(0.8-6.2)$ & 0.15 & $\mathrm{NI}$ & $\mathrm{NI}$ \\
\hline Oceania & 6 & 0 & 0 & 6 & 100 & NA & NA & $\mathrm{NI}$ & $\mathrm{NI}$ \\
\hline Europe & 524 & 13 & 2.5 & 511 & 97.5 & Ref & Ref & $\mathrm{NI}$ & $\mathrm{NI}$ \\
\hline \multicolumn{7}{|l|}{ Duration of hospitalisation } & 0.03 & NA & \\
\hline $1-2$ days & 83 & 0 & 0 & 83 & 100 & NA & NA & $\mathrm{NI}$ & $\mathrm{NI}$ \\
\hline 3-7 days & 208 & 3 & 1.4 & 205 & 98.6 & $0.2(0.0-.0 .6)$ & $<0.01$ & $\mathrm{NI}$ & $\mathrm{NI}$ \\
\hline $8-14$ days & 186 & 6 & 3.2 & 180 & 96.8 & $0.4(0.2-1.0)$ & 0.06 & $\mathrm{NI}$ & $\mathrm{NI}$ \\
\hline Over 14 days & 193 & 15 & 7.8 & 178 & 92.2 & Ref & Ref & $\mathrm{NI}$ & $\mathrm{NI}$ \\
\hline Data missing & 28 & 1 & 3.6 & 27 & 96.4 & $\mathrm{NI}$ & $\mathrm{NI}$ & $\mathrm{NI}$ & $\mathrm{NI}$ \\
\hline \multicolumn{10}{|l|}{ ICU treatment abroad } \\
\hline Yes & 190 & 12 & 6.3 & 178 & 93.7 & $2.6(1.2-5.7)$ & 0.02 & $\mathrm{NI}$ & $\mathrm{NI}$ \\
\hline No & 508 & 13 & 2.6 & 495 & 97.4 & Ref & Ref & $\mathrm{NI}$ & $\mathrm{NI}$ \\
\hline \multicolumn{10}{|c|}{ Major invasive procedure abroad } \\
\hline Yes & 290 & 14 & 4.8 & 276 & 95.2 & $1.8(0.8-4.1)$ & 0.14 & $\mathrm{NI}$ & $\mathrm{NI}$ \\
\hline No & 408 & 11 & 2.7 & 397 & 97.3 & Ref & Ref & $\mathrm{NI}$ & $\mathrm{NI}$ \\
\hline \multicolumn{10}{|l|}{ Antibiotic use abroad } \\
\hline Yes & 383 & 19 & 5.0 & 364 & 95.0 & $2.7(1.1-6.8)$ & 0.03 & $2.5(1.0-6.5)$ & 0.05 \\
\hline No & 315 & 6 & 1.9 & 309 & 98.1 & Ref & Ref & Ref & Ref \\
\hline \multicolumn{10}{|l|}{ Reason for hospitalisation } \\
\hline Trauma & 323 & 11 & 3.4 & 312 & 96.6 & Ref & Ref & $\mathrm{NI}$ & $\mathrm{NI}$ \\
\hline Non-trauma & 375 & 14 & 3.7 & 361 & 96.3 & $1.1(0.5-2.5)$ & 0.82 & $\mathrm{NI}$ & $\mathrm{NI}$ \\
\hline Antibiotic use during MRSA & eening & & & & & & & & \\
\hline Yes & 185 & 9 & 4.9 & 176 & 95.1 & $1.7(0.7-4.0)$ & 0.22 & $\mathrm{NI}$ & $\mathrm{NI}$ \\
\hline No & 477 & 14 & 2.9 & 463 & 97.1 & Ref & Ref & $\mathrm{NI}$ & $\mathrm{NI}$ \\
\hline Data missing & 36 & 2 & 5.6 & 34 & 94.4 & $\mathrm{NI}$ & $\mathrm{NI}$ & $\mathrm{NI}$ & $\mathrm{NI}$ \\
\hline
\end{tabular}

AOR: adjusted odds ratio; $\mathrm{CCl}$ : Charlson Comorbidity Index; $\mathrm{Cl}$ : confidence interval; ICU: intensive care unit; NA: not applicable; NI: not included; OR: odds ratio; Ref: reference; VFR: visiting friends and relatives.

a Due to a low number of MRSA positive cases $(n=25)$, only the following variables were included in the multivariable model: chronic alcohol abuse, antibiotic use abroad. 
patients, MDR bacteria from non-screened anatomic sites, such as skin lesions, could have been missed. However, we believe that the strict inclusion criteria for triple-site MRSA and faecal MDRGNB screenings together with clear hospital guidelines have resulted in a realistic yield.

With regards to antibiotic treatments, records from hospitals abroad were often not available, and antibiotics in use at the time of screening may have affected MDR bacterial findings. In general, depending on the setting, a concomitant antimicrobial effect may lead to unsuccessful bacterial culture or, contrarily, a selection of resistant strains. The complex effects of various antibiotics and their combinations could not be analysed. Finally, the rate of symptomatic MDR bacterial infections may be an underestimate, since infections without microbiological verification were not covered. As infections treated abroad and those diagnosed after discharge (or 30 days) were not recorded, a different design would be needed to evaluate the total burden of MDR bacterial infections among this patient population.

\section{Conclusions}

Colonisation by MDR bacteria is common among patients transferred from hospitals in high-prevalence countries. The most prevalent bacteria, ESBL-PE, are also frequently carried by non-hospitalised travellers. In addition, a substantial number of non-ESBL-PE strains, such as carbapenemase-producing bacteria, was detected. Among the variety of risk factors of MDR bacterial colonisation that were identified, geographical region of hospitalisation proved the strongest predictor of MDR findings. The study indicates that systematic screening of international transfer patients is warranted; our data serve as valuable background for devising infection control policies.

\section{Acknowledgements}

We wish to thank Jukka Ollgren (Finnish Institute for Health and Welfare, THL) for advice on statistical analyses.

Funding: This work was supported by the Finnish Governmental Subsidy for Health Science Research, The Sigrid Jusélius Foundation and The Finnish Association of Infectious diseases. The funding bodies were not involved in study design, in the data collection, analysis or interpretation of data, writing of the manuscript nor the decision to submit for publication.

\section{Conflict of interest}

MK has participated in a conference sponsored by Astellas Pharma. JK is a shareholder in Orion Corporation. AK has received investigator-initiated grants (Valneva, Pfizer) and on an individual occasion consulted an advisory board (Valneva). None of the interests listed are relevant to the current manuscript. TK and HAM report no potential conflicts of interest.
Authors' contributions

Study concept and design: MK, AK; data collection: MK, TK, JK, HM; statistics: MK; drafting of manuscript MK, AK; critical comments on manuscript: TK, JK, HM; approving final manuscript: all.

\section{References}

1. Woerther PL, Andremont A, Kantele A. Travel-acquired ESBL-producing Enterobacteriaceae: impact of colonization at individual and community level. J Travel Med.

2017;24(suppl_1):S29-s34. https://doi.org/10.1093/jtm/taw101 PMID: 28520999

2. de Kraker ME, Davey PG, Grundmann H, BURDEN study group. Mortality and hospital stay associated with resistant Staphylococcus aureus and Escherichia coli bacteremia: estimating the burden of antibiotic resistance in Europe. PLoS Med. 2011;8(10):e1001104. https://doi.org/10.1371/journal. pmed.1001104 PMID: 22022233

3. Prematunge C, MacDougall C, Johnstone J, Adomako K, Lam $F$, Robertson J, et al. VRE and VSE bacteremia outcomes in the era of effective VRE therapy: a systematic review and metaanalysis. Infect Control Hosp Epidemiol. 2016;37(1):26-35. https://doi.org/10.1017/ice.2015.228 PMID: 26434609

4. Kantele A, Lääveri T, Mero S, Häkkinen IMK, Kirveskari J, Johnston BD, et al. Despite predominance of uropathogenic/ extraintestinal pathotypes among travel-acquired Extendedspectrum $\beta$-Lactamase-producing Escherichia coli, the most commonly associated clinical manifestation is travelers' diarrhea. Clin Infect Dis. 2020;70(2):210-8. https://doi. org/10.1093/cid/ciz182 PMID: 31034006

5. Fischer D, Veldman A, Schäfer V, Diefenbach M. Bacterial colonization of patients undergoing international air transport: a prospective epidemiologic study. J Travel Med. 2004;11(1):448. https://doi.org/10.2310/7060.2004.13647 PMID: 14769287

6. Kaiser AM, Schultsz C, Kruithof GJ, Debets-Ossenkopp Y, Vandenbroucke-Grauls C. Carriage of resistant microorganisms in repatriates from foreign hospitals to The Netherlands. Clin Microbiol Infect. 2004;10(11):972-9. https://doi.org/10.1111/ j.1469-0691.2004.01000.x PMID: 15521999

7. Nemeth J, Ledergerber B, Preiswerk B, Nobile A, Karrer S, Ruef $C$, et al. Multidrug-resistant bacteria in travellers hospitalized abroad: prevalence, characteristics, and influence on clinical outcome. J Hosp Infect. 2012;82(4):254-9. https://doi. org/10.1016/j.jhin.2012.08.017 PMID: 23103249

8. Josseaume J, Verner L, Brady WJ, Duchateau FX. Multidrug resistant bacteria among patients treated in foreign hospitals: management considerations during medical repatriation. J Travel Med. 2013;20(1):22-8. https://doi.org/10.1111/j.17088305.2012.00668.x PMID: 23279227

9. Birgand G, Armand-Lefevre L, Lepainteur M, Lolom I, Neulier $C$, Reibel F, et al. Introduction of highly resistant bacteria into a hospital via patients repatriated or recently hospitalized in a foreign country. Clin Microbiol Infect. 2014;20(11):0887-90. https://doi.org/10.1111/1469-0691.12604 PMID: 25069719

10. Angue M, Allou N, Belmonte O, Lefort Y, Lugagne N, Vandroux $\mathrm{D}$, et al. Risk factors for colonization with multidrug-resistant bacteria among patients admitted to the intensive care unit after returning from abroad. J Travel Med. 2015;22(5):300-5. https://doi.org/10.1111/jtm.12220 PMID: 26081076

11. Kaspar T, Schweiger A, Droz S, Marschall J. Colonization with resistant microorganisms in patients transferred from abroad: who needs to be screened? Antimicrob Resist Infect Control. 2015;4(1):31. https://doi.org/10.1186/s13756-015-0071-6 PMID: 26213620

12. Mutters NT, Günther F, Sander A, Mischnik A, Frank U. Influx of multidrug-resistant organisms by country-to-country transfer of patients. BMC Infect Dis. 2015;15(1):466. https://doi. org/10.1186/s12879-015-1173-8 PMID: 26508038

13. Khawaja T, Kirveskari J, Johansson S, Vaisanen J, Djupsjobacka A, Nevalainen A, et al. Patients hospitalized abroad as importers of multiresistant bacteria-a cross-sectional study. Clin Microbiol Infect. 2017;23(9):673.e1-.e8. https://doi. org/10.1016/j.cmi.2017.02.003 PMID: 28196696

14. Charlson ME, Pompei P, Ales KL, MacKenzie CR. A new method of classifying prognostic comorbidity in longitudinal studies: development and validation. J Chronic Dis. 1987;40(5):373-83. https://doi.org/10.1016/0021-9681(87)90171-8 PMID: 3558716

15. Aro T, Kantele A. High rates of meticillin-resistant Staphylococcus aureus among asylum seekers and refugees admitted to Helsinki University Hospital, 2010 to 2017. Euro Surveill. 2018;23(45). https://doi.org/10.2807/1560-7917. ES.2018.23.45.1700797 PMID: 30424828 
16. The European Committee on Antimicrobial Susceptibility Testing (EUCAST). Breakpoint tables for interpretation of MICs and zone diameters, versions 1.3-9.0 (2011-2019). Växjö: EUCAST; 2020. Available from: https://eucast.org/ ast_of_bacteria/previous_versions_of_documents

17. Clinical and Laboratory Standards Institute. Performance standards for antimicrobial susceptibility testing, 19th informational supplement M100-S19. Wayne: CLSI; 2009.

18. Arcilla MS, van Hattem JM, Haverkate MR, Bootsma MCJ, van Genderen PJJ, Goorhuis A, et al. Import and spread of extended-spectrum $\beta$-lactamase-producing Enterobacteriaceae by international travellers (COMBAT study): a prospective, multicentre cohort study. Lancet Infect Dis. 2017;17(1):7885. https://doi.org/10.1016/S1473-3099(16)30319-X PMID: 27751772

19. Tängdén T, Cars O, Melhus A, Löwdin E. Foreign travel is a major risk factor for colonization with Escherichia coli producing CTX-M-type extended-spectrum beta-lactamases: a prospective study with Swedish volunteers. Antimicrob Agents Chemother. 2010;54(9):3564-8. https://doi.org/10.1128/ AAC.00220-10 PMID: 20547788

20. Paltansing S, Vlot JA, Kraakman ME, Mesman R, Bruijning ML, Bernards AT, et al. Extended-spectrum $\beta$-lactamase-producing enterobacteriaceae among travelers from the Netherlands. Emerg Infect Dis. 2013;19(8):1206-13. https://doi.org/10.3201/ eid1908.130257 PMID: 23885972

21. Ruppé E, Armand-Lefèvre L, Estellat C, El-Mniai A, Boussadia $\mathrm{Y}$, Consigny $\mathrm{PH}$, et al. Acquisition of carbapenemase-producing Enterobacteriaceae by healthy travellers to India, France, February 2012 to March 2013. Euro Surveill. 2014;19(14):20768. https://doi.org/10.2807/1560-7917.ES2014.19.14.20768 PMID: 24739981

22. ÖstholmBalkhed $\AA$, Tärnberg M, Nilsson M, Nilsson LE, Hanberger H, Hällgren A, Southeast Sweden Travel Study Group. Duration of travel-associated faecal colonisation with ESBL-producing Enterobacteriaceae - A one year followup study. PLoS One. 2018;13(10):e0205504. https://doi. org/10.1371/journal.pone.0205504 PMID: 30356258

23. Schaumburg F, Sertic SM, Correa-Martinez C, Mellmann A, Kock R, Becker K. Acquisition and colonization dynamics of antimicrobial-resistant bacteria during international travel: a prospective cohort study. Clin Microbiol Infect. 2019;25(10):1287.e1-.e7. https://doi.org/10.1016/j. cmi.2019.03.002 PMID: 30898722

24. Chanderraj R, Millar JA, Patel TS, Read AF, Washer L, Kaye $\mathrm{KS}$, et al. Vancomycin-resistant Enterococcus acquisition in a tertiary care hospital: testing the roles of antibiotic use, proton pump inhibitor use, and colonization pressure. Open Forum Infect Dis. 2019;6(4):ofz139. https://doi.org/10.1093/ofid/ ofz139 PMID: 31024976

25. Falagas ME, Kopterides P. Risk factors for the isolation of multi-drug-resistant Acinetobacter baumannii and Pseudomonas aeruginosa: a systematic review of the literature. J Hosp Infect. 2006;64(1):7-15. https://doi. org/10.1016/j.jhin.2006.04.015 PMID: 16822583

26. Tacconelli E, De Angelis G, Cataldo MA, Pozzi E, Cauda R. Does antibiotic exposure increase the risk of methicillin-resistant Staphylococcus aureus (MRSA) isolation? A systematic review and meta-analysis. J Antimicrob Chemother. 2008;61(1):26-38. https://doi.org/10.1093/jac/dkm416 PMID: 17986491

27. Kantele A, Lääveri T, Mero S, Vilkman K, Pakkanen SH, Ollgren J, et al. Antimicrobials increase travelers' risk of colonization by extended-spectrum betalactamase-producing Enterobacteriaceae. Clin Infect Dis. 2015;60(6):837-46. https://doi.org/10.1093/cid/ciu957 PMID: 25613287

28. Ny S, Kozlov R, Dumpis U, Edquist P, Gröndahl-Yli-Hannuksela $\mathrm{K}$, Kling AM, et al. , NoDARS ESBL-carrier working group. Large variation in ESBL-producing Escherichia coli carriers in six European countries including Russia. Eur J Clin Microbiol Infect Dis. 2018;37(12):2347-54. https://doi.org/10.1007/s10096018-3382-8 PMID: 30338465

29. Ruppé E, Armand-Lefèvre L, Estellat C, Consigny PH, El Mniai A, Boussadia $Y$, et al. High rate of acquisition but short duration of carriage of multidrug-resistant Enterobacteriaceae after travel to the tropics. Clin Infect Dis. 2015;61(4):593-600. https://doi.org/10.1093/cid/civ333 PMID: 25904368

30. Räisänen K, Jalava J. Bakteerien mikrobilääkeresistenssi Suomessa: Finres 2018. [Antimicrobial resistance of bacteria in Finland: Finres 2018]. Finnish Institute for Health and Welfare. 2019. Available from: http://www.julkari.fi/ handle/10024/138818. Finish.

31. HUSRES HUSRES Herkkyystilastot 2010-2018. [HUSRES Annual reports 2010-2018]. Available from: https://huslab.fi/ ohjekirjan_liitteet/mikrobiologian_ohjeet/mikrobiologian_ tilastoja/index.html. Finnish.

32. Moore C, Dhaliwal J, Tong A, Eden S, Wigston C, Willey B, et al. Risk factors for methicillin-resistant Staphylococcus aureus (MRSA) acquisition in roommate contacts of patients colonized or infected with MRSA in an acute-care hospital. Infect Control Hosp Epidemiol. 2008;29(7):600-6. https://doi. org/10.1086/588567 PMID: 18624667

33. Reuland EA, Sonder GJ, Stolte I, Al Naiemi N, Koek A, Linde $\mathrm{GB}$, et al. Travel to Asia and traveller's diarrhoea with antibiotic treatment are independent risk factors for acquiring ciprofloxacin-resistant and extended spectrum $\beta$-lactamaseproducing Enterobacteriaceae-a prospective cohort study. Clin Microbiol Infect. 2016;22(8):731.e1-7. https://doi. org/10.1016/j.cmi.2016.05.003 PMID: 27223840

34. van Hattem JM, Arcilla MS, Bootsma MC, van Genderen PJ, Goorhuis A, Grobusch MP, et al. Prolonged carriage and potential onward transmission of carbapenemase-producing Enterobacteriaceae in Dutch travelers. Future Microbiol. 2016;11(7):857-64. https://doi.org/10.2217/fmb.16.18 PMID: 27357522

35. Stenhem M, Ortqvist A, Ringberg H, Larsson L, Olsson Liljequist B, Haeggman S, et al. Imported methicillinresistant Staphylococcus aureus, Sweden. Emerg Infect Dis. 2010;16(2):189-96. https://doi.org/10.3201/eid1602.081655 PMID: 20113546

36. Kantele A, Kuenzli E, Dunn SJ, Dance DAB, Newton PN, Davong $V$, et al. Dynamics of intestinal multidrug-resistant bacteria colonisation contracted by visitors to a high-endemic setting: a prospective, daily, real-time sampling study. Lancet Microbe. 2021;2(4):e151-8. https://doi.org/10.1016/S26665247(20)30224-X PMID: 33821248

\section{License, supplementary material and copyright}

This is an open-access article distributed under the terms of the Creative Commons Attribution (CC BY 4.0) Licence. You may share and adapt the material, but must give appropriate credit to the source, provide a link to the licence and indicate if changes were made.

Any supplementary material referenced in the article can be found in the online version.

This article is copyright of the authors or their affiliated institutions, 2021. 